\title{
Chronic Constrictive Pericarditis in Association with End-Stage Renal Disease
}

\author{
Roman L. Kleynberg, ${ }^{1}$ Vera M. Kleynberg, ${ }^{2}$ Leonid M. Kleynberg, ${ }^{3}$ \\ and Danny Farahmandian ${ }^{2}$ \\ ${ }^{1}$ Department of Internal Medicine, Olive View-UCLA Medical Center, 14445 Olive View Drive, Sylmar, CA 91342-1438, USA \\ ${ }^{2}$ Department of Internal Medicine, Encino-Tarzana Regional Medical Center, 16237 Ventura Boulevard, CA 91436-2201, USA \\ ${ }^{3}$ Department of Internal Medicine, Brotman Medical Center, 3828 Delmas Terrace, Culver City, CA 90232-6806, USA
}

Correspondence should be addressed to Roman L. Kleynberg, roman.kleynberg@ucla.edu

Received 8 November 2010; Revised 12 February 2011; Accepted 22 February 2011

Academic Editor: Jaime Uribarri

Copyright (c) 2011 Roman L. Kleynberg et al. This is an open access article distributed under the Creative Commons Attribution License, which permits unrestricted use, distribution, and reproduction in any medium, provided the original work is properly cited.

Pericardial involvement in end-stage renal disease (ESRD) is manifested most commonly as acute uremic or dialysis pericarditis and infrequently as chronic constrictive pericarditis (CCP). We report a 27 -year-old patient with a history of uncontrolled hypertension, end-stage-renal disease on hemodialysis, who presented with recurrent ascites, dyspnea, and hypotension. After diagnosis with CCP, a partial pericardiectomy was performed; however, the patient did not improve and a salvage total pericardiectomy soon followed. He continued to decompensate and expired following a terminal extubation. No definitive cause of constrictive pericarditis was found. Nonetheless, we surmise it may have developed secondary to his end-stage renal disease. A literature review revealed end-stage kidney disease as a relatively uncommon cause of CCP; only a few other such associations have thus far been reported.

\section{Introduction}

The pericardium is an avascular fibrotic bag that envelops the heart. It is composed of two layers: the serous and fibrous pericardium. The serous layer is also divided into two parts, the parietal pericardium which is intimately fused to the fibrous pericardium and also the visceral pericardium which is a part of the epicardium. In between the visceral and parietal pericardium is a thin potential space consisting of 20-30 cc of serous fluid that fills a potential space known as the pericardial cavity. In the case of CCP, the fibrotic pericardium thickens and forms a noncompliant casing surrounding the heart. This prevents the heart from expanding as blood enters the ventricles during diastole. Pericardial involvement in end-stage renal disease (ESRD) is manifested most commonly as acute uremic or dialysis pericarditis and infrequently as chronic constrictive pericarditis. The cause of $60 \%$ of cases of CCP is unknown and is labeled as idiopathic [1]. Moreover, CCP is commonly confused with other diagnoses such as end-stage-liver failure, idiopathic cardiomyopathy, and restrictive cardiomyopathy. In our case presentation, we report an advanced case of constrictive pericarditis associated with end-stage-renal disease and follow with a literature review.

\section{Case Report}

A 27-year-old Armenian man with a history of hypertension, end-stage-renal disease on hemodialysis, and recently diagnosed CCP presented to our hospital with massive ascites, dyspnea, and hypotension. He has had ESRD over the past three years, the cause of which was most likely secondary to uncontrolled hypertension. His social history included a 1 pack-year of tobacco smoking and no alcohol or recreational drug use. On physical exam, his vitals were $80-90 \mathrm{~s} / 30$ $40 \mathrm{~s}$ and he had decreased breath sounds on the left side, decreased heart sounds, some crackles diffusely, and 2+ pitting edema diffusely over the lower extremities. Over the past few months, he had developed massive ascites, and a through workup was undertaken to discover the underlying 
cause of his symptoms which included intrahepatic pressure measurements, cholecystectomy, appendectomy, liver biopsy, and a peritoneal biopsy. In the hospital, he was dialyzed aggressively and received multiple paracentesis (the first of which drained 6 liters of greenish fluid), but the ascites continued to return in 1-2 days. An echocardiogram showed normal left ventricular systolic function, EF 55\%, dilated left and right atrium, and evidence of pressure and volume overload over the right ventricle (right ventricular hypertrophy). A CT scan showed pericardial thickening and bilateral pleural effusions (Figure 1).

A left and right heart cardiac catheterization demonstrated a thick peal around the heart, ejection fraction of $55 \%$, PAP of $30 \mathrm{~mm} \mathrm{Hg}$, LVED pressures of $30-35 \mathrm{~mm} \mathrm{Hg}$, and also equalization of pressures on both the right and left side of the heart (Figure 2).

The patient had never in prior had any episodes of acute pericarditis. Following the diagnosis of CCP, a partial pericardiectomy was performed; however, the patient did not improve and a salvage total pericardiectomy soon followed. At sternotomy, the pericardium was measured to be $5-6 \mathrm{~mm}$ in thickness. A postsurgery catheterization showed persistent constrictive pericarditis. No specific etiology for CCP was found after numerous histopathological, serological, and bacteriological studies including CBC with differential, hepatocellular function tests, ESR, CRP, BNP, ANA, RF, ASO titer, anti-DNA Abs, PPD test, HIV ELISA, thryroid studies, pericardial fluid analysis ( $\mathrm{LDH}$, total protein, cell count, glucose, gram stain, and cultures), and cardiac biomarkers (troponin I). His labs did not reveal any evidence of hyperparathyroidism; on presentation calcium was $9.5 \mathrm{mEq} / \mathrm{dL}$, phosphorus $3.8 \mathrm{mg} / \mathrm{dL}$, PTH was 45 , and patient was not on any Vitamin D analog medication. Histology of the pericardial layer was not obtained. The patient developed complications from the total pericardiectomy including septic shock, and shortly thereafter expired following a terminal extubation.

\section{Discussion}

The most common cause of constrictive pericarditis (up to $45-55 \%$ of cases) is idiopathic or viral in etiology [24]. The most common identifiable causes include postcardiac surgery $(37 \%)$, pericarditis (16-20\%), and mediastinal radiation $(9 \%)[2,3,5]$. Other recognized but less common causes include connective tissue disorders (such as RA and SLE), malignancy (especially breast cancer, lung cancer and lymphoma), and local trauma. Tuberculosis was once recognized as the most common cause of constrictive pericarditis and continues to be a major problem in the developing world. Other causes include purulent infections, histoplasmosis, and chronic renal failure treated by chronic dialysis [6]. In the case of our patient, no specific etiology was found after numerous histopathological, serological and bacteriological exams. Though undetected viral cause and the large majority of constrictive pericarditis, because of his ESRD and recent initiation of HD just a few months prior to his presentation, we believe the ESRD is the underlying etiology of his constrictive pericarditis. The pericardium

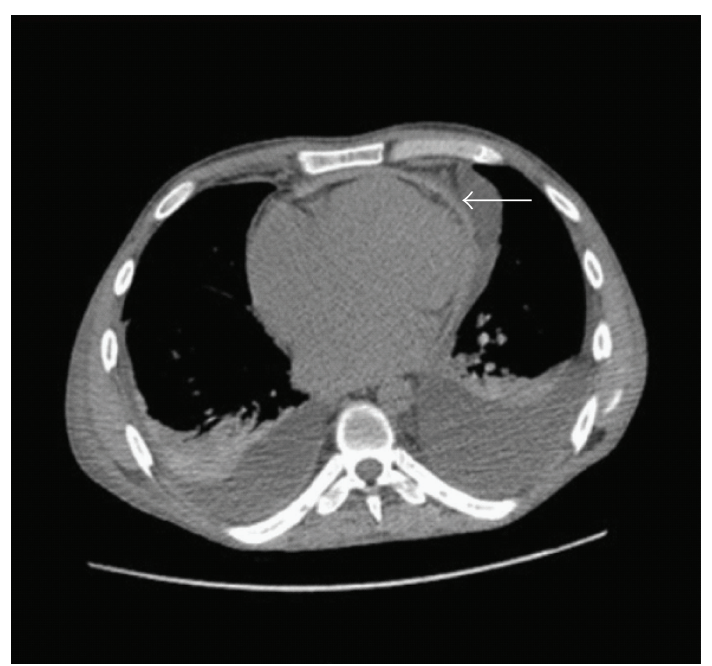

FIgURE 1: A thorax computerized tomographic scan (without contrast) revealing bilateral pleural effusions and pericardial thickening (arrow) of both ventricles that produce constriction.

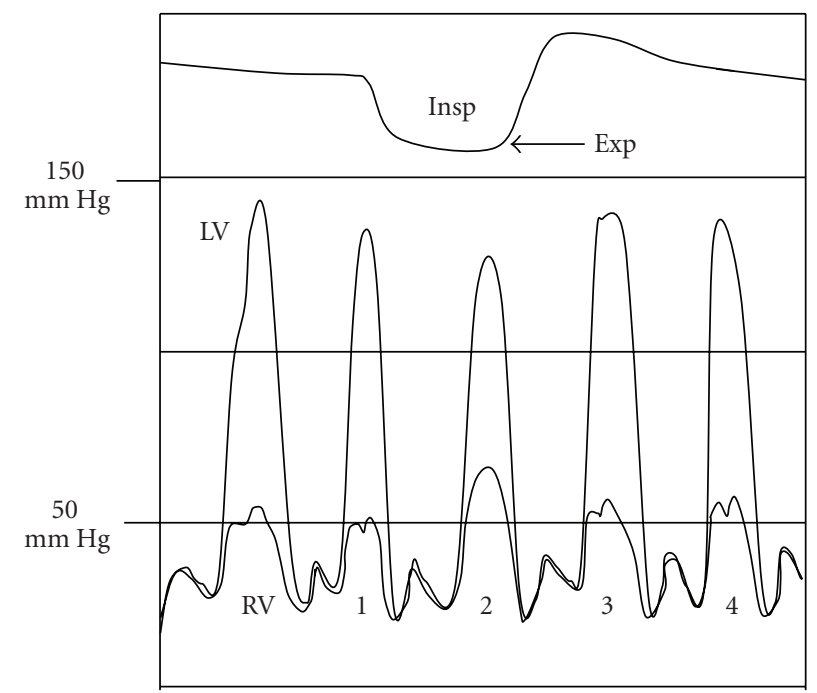

FIGURE 2: Simultaneous left ventricular (LV) and right ventricular (RV) systolic pressures in a patient with constrictive pericarditis. There is a decrease in the LV pressure and a concomitant increase in the RV pressure during peak inspiration (arrow) during the four beats that are plotted of the respiratory cycle-also known as "pulses paradoxus." Also, equalization of end-diastolic pressures is observed in both ventricles. Note the "square root sign" or "dip and plateau sign" of the left ventricular waveforms, which confirm the diagnosis of constrictive pericarditis. Exp, expiration; Insp, inspiration.

in ESRD patients commonly will present as an acute uremic or dialysis pericarditis and less commonly as chronic constrictive pericarditis [7]. Following a MEDLINE literature search, we were only able to find a few such association $[5,8,9]$ between CCP and ESRD when using the keywords: "renal failure and constrictive pericarditis" and "ESRD and pericarditis." Thus, end-stage-renal disease should be considered in the underlying etiology of constrictive pericarditis. 
Constrictive pericarditis is a rare disorder that manifests predominantly as right heart failure and, in severe cases, systemic hypotension and circulatory collapse [10]. The diagnosis can often be a challenge to diagnose [11]. Some common clinical features include edema, ascites, raised jugular venous pressure, pleural effusion, and hepatomegaly, all commonly found with right-heart strain. Around 1/3 of patients with constrictive pericarditis have coexistent atrial fibrillation [12]. On imaging, a plain chest radiography may show pericardial calcification and unexplained pleural effusions especially in severe stages of the disease [11]. Echocardiography may validate the presence of small ventricular dimensions with preserved systolic function, and dilated atria, all findings present in our patient. Abrupt termination of diastolic filling may show a characteristic "septal bounce" [13]. Cardiac catheterization studies allow for definitive confirmation.

Constrictive pericarditis may be differentiated from other forms of heart failure such as restrictive cardiomyopathy by the presentation of thickened pericardium on computed tomography and magnetic resonance imaging [14]. Localized fibrosis and intrapericardial calcifications are some of the histopathological features often associated with constrictive pericarditis. During early diastole, ventricular filling is rapid due to high systemic venous pressure which results in the elevation and equilibration of the diastolic pressures of all four heart chambers. However, late diastolic filling is inhibited by the rigid pericardium; it is restricted as the intracardiac volume reaches the limit set by the noncompliant pericardium. This acts in general to reduce total cardiac output. Our case was an exception to this rule as cardiac output was preserved, at least in part, most likely due to the high end diastolic filling pressure or LVEDP (measured to be equal on both the right and left ventricle at $30 \mathrm{~mm} \mathrm{Hg}$ ).

The definitive treatment of constrictive pericarditis is total pericardiectomy and aggressive treatment of any underlying disease process. Total rather than partial pericardiectomy yields better outcome and should be undertaken as early as possible which has been shown to yield a better clinical outcome [15]. Also, survival is dependent on the underlying etiology, and patients with idiopathic constrictive pericarditis usually have the best outcome following pericardiectomy [3]. The 30-day mortality after pericardiectomy was $14 \%$ in a historical cohort. Long-term survival ranged from $78 \%$ at 5 years, $57 \%$ at 10 years, and limited by severe preoperative functional disability, renal failure, and nonresectable myocardial calcifications [2]. Our unfortunate patient had many of these risk factors and survived just 2 weeks following his total pericardiectomy. Thus, in patients with end-stage-renal disease, chronic indolent or subclinical dialysis associated with pericarditis may lead to constrictive pericarditis.

\section{Conclusion}

Both the etiology and physiology of constrictive pericarditis are reviewed and it is of value for clinicians to consider ESRD as an underlying cause in their workup of constrictive pericarditis after first ruling out other more common etiologies.

\section{Funding}

No funding was provided for the research/writing of this paper.

\section{References}

[1] J. C. Dos Santos Guitti, "Constrictive pericarditis in a 19month-old child," Arquivos Brasileiros de Cardiologia, vol. 74, no. 1, pp. 51-54, 2000.

[2] L. H. Ling, J. K. Oh, H. V. Schaff et al., "Constrictive pericarditis in the modern era: evolving clinical spectrum and impact on outcome after pericardiectomy," Circulation, vol. 100, no. 13, pp. 1380-1386, 1999.

[3] S. C. Bertog, S. K. Thambidorai, K. Parakh et al., "Constrictive pericarditis: etiology and cause-specific survival after pericardiectomy," Journal of the American College of Cardiology, vol. 43, no. 8, pp. 1445-1452, 2004.

[4] J. Cameron, S. N. Oesterle, J. C. Baldwin, and E. W. Hancock, "The etiologic spectrum of constrictive pericarditis," American Heart Journal, vol. 113, no. 2, part 1, pp. 354-360, 1987.

[5] C. B. Marcu, E. Caracciolo, and T. Donohue, "Rapid of progression of pericardial calcification in a patient with end-stage renal disease," Catheterization and Cardiovascular Interventions, vol. 65, no. 1, pp. 43-46, 2005.

[6] E. Braunwald, "Pericardial disease," in Harrison's Principles of Internal Medicine, A. S. Fauci, E. Braunwald, K. J. Isselbacher et al., Eds., vol. 1, pp. 1334-1340, McGraw-Hill, New York, NY, USA, 1998.

[7] M. A. Alpert and M. D. Ravenscraft, "Pericardial involvement in end-stage renal disease," American Journal of the Medical Sciences, vol. 325, no. 4, pp. 228-236, 2003.

[8] N. Hateboer, R. J. McGonigle, and C. T. Lewis, "Pericardiectomy after two decades of constrictive pericarditis in a patient with chronic renal failure," Nephrology Dialysis Transplantation, vol. 10, no. 10, pp. 1935-1937, 1995.

[9] A. M. Sloan, "Uremic constrictive pericarditis," The Medical annals of the District of Columbia, vol. 43, no. 1, pp. 1-3, 1974.

[10] C. R. Asher and A. L. Klein, "Diastolic heart failure: restrictive cardiomyopathy, constrictive pericarditis, and cardiac tamponade: clinical and echocardiographic evaluation," Cardiology in Review, vol. 10, no. 4, pp. 218-229, 2002.

[11] M. W. Akhter, I. N. Nuño, and S. H. Rahimtoola, "Constrictive pericarditis masquerading as chronic idiopathic pleural effusion: importance of physical examination," American Journal of Medicine, vol. 119, no. 7, pp. e1-e4, 2006.

[12] T. Tabata, S. S. Kabbani, R. D. Murray, J. D. Thomas, I. Abdalla, and A. L. Klein, "Difference in the respiratory variation between pulmonary venous and mitral inflow doppler velocities in patients with constrictive pericarditis with and without atrial fibrillation," Journal of the American College of Cardiology, vol. 37, no. 7, pp. 1936-1942, 2001.

[13] R. B. Himelman, E. Lee, and N. B. Schiller, "Septal bounce, vena cava plethora, and pericardial adhesion: informative two-dimensional echocardiographic signs in the diagnosis of pericardial constriction," Journal of the American Society of Echocardiography, vol. 1, no. 5, pp. 333-340, 1988. 
[14] T. Masui, S. Finck, and C. B. Higgins, "Constrictive pericarditis and restrictive cardiomyopathy: evaluation with MR imaging," Radiology, vol. 182, no. 2, pp. 369-373,1992.

[15] U. K. Chowdhury, G. K. Subramaniam, A. S. Kumar et al., "Pericardiectomy for constrictive pericarditis: a clinical, echocardiographic, and hemodynamic evaluation of two surgical techniques," Annals of Thoracic Surgery, vol. 81, no. 2, pp. 522-529, 2006. 


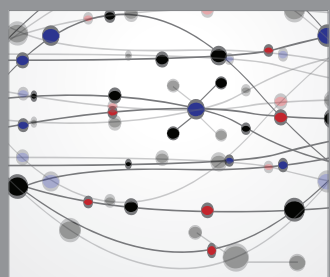

The Scientific World Journal
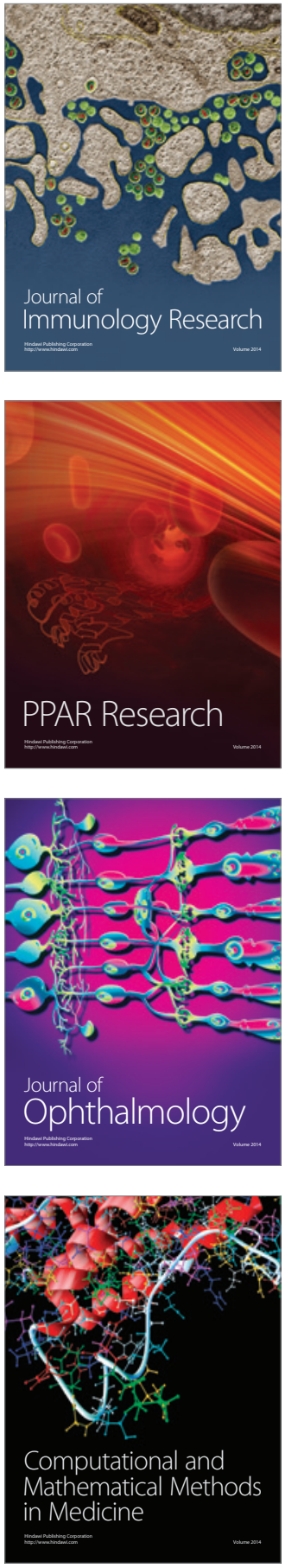

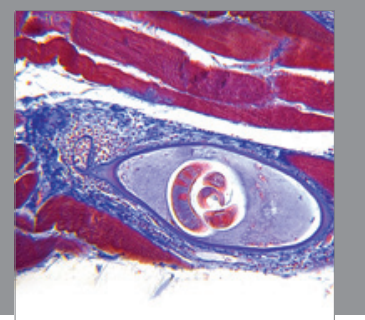

Gastroenterology

Research and Practice
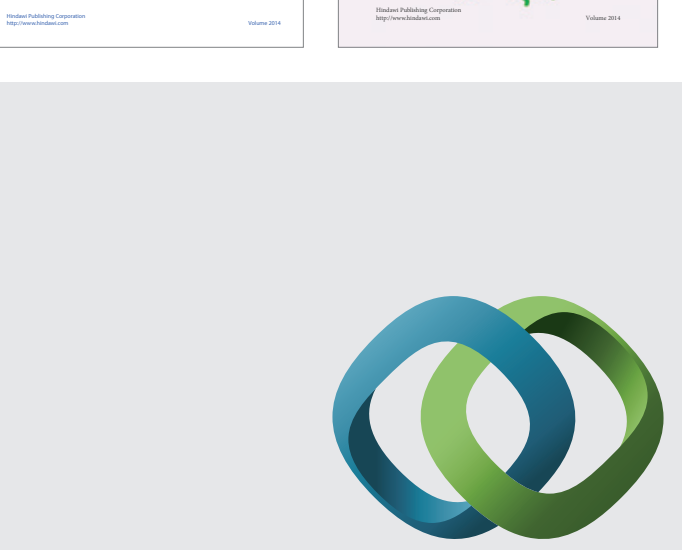

\section{Hindawi}

Submit your manuscripts at

http://www.hindawi.com
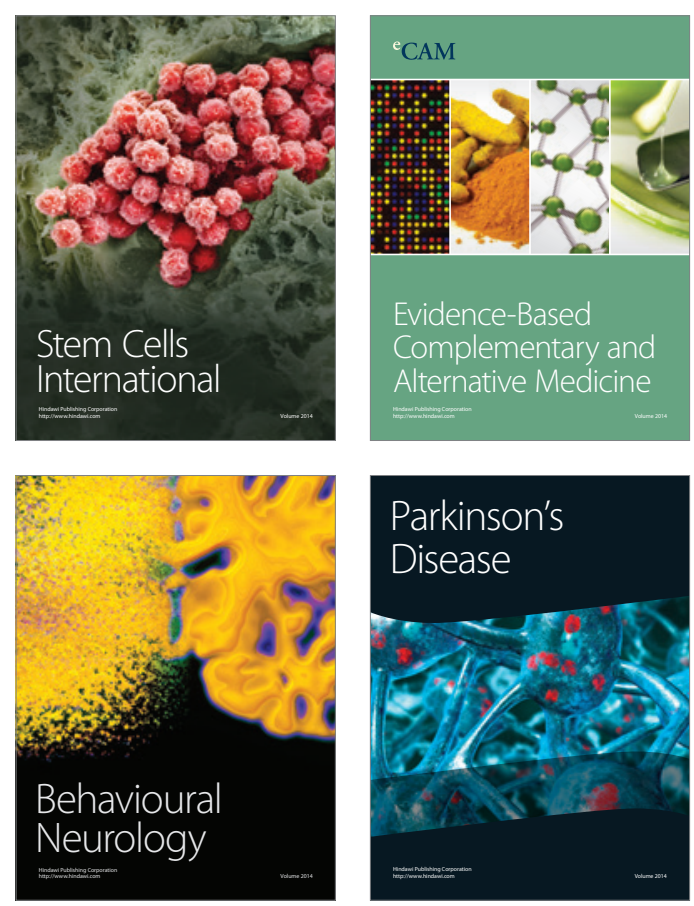

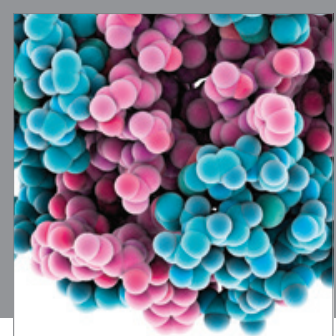

Journal of
Diabetes Research

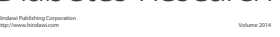

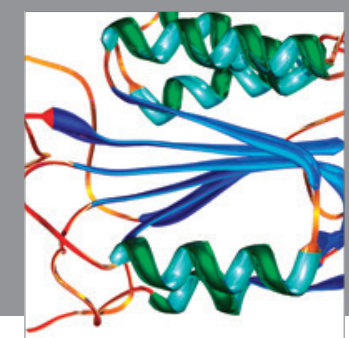

Disease Markers
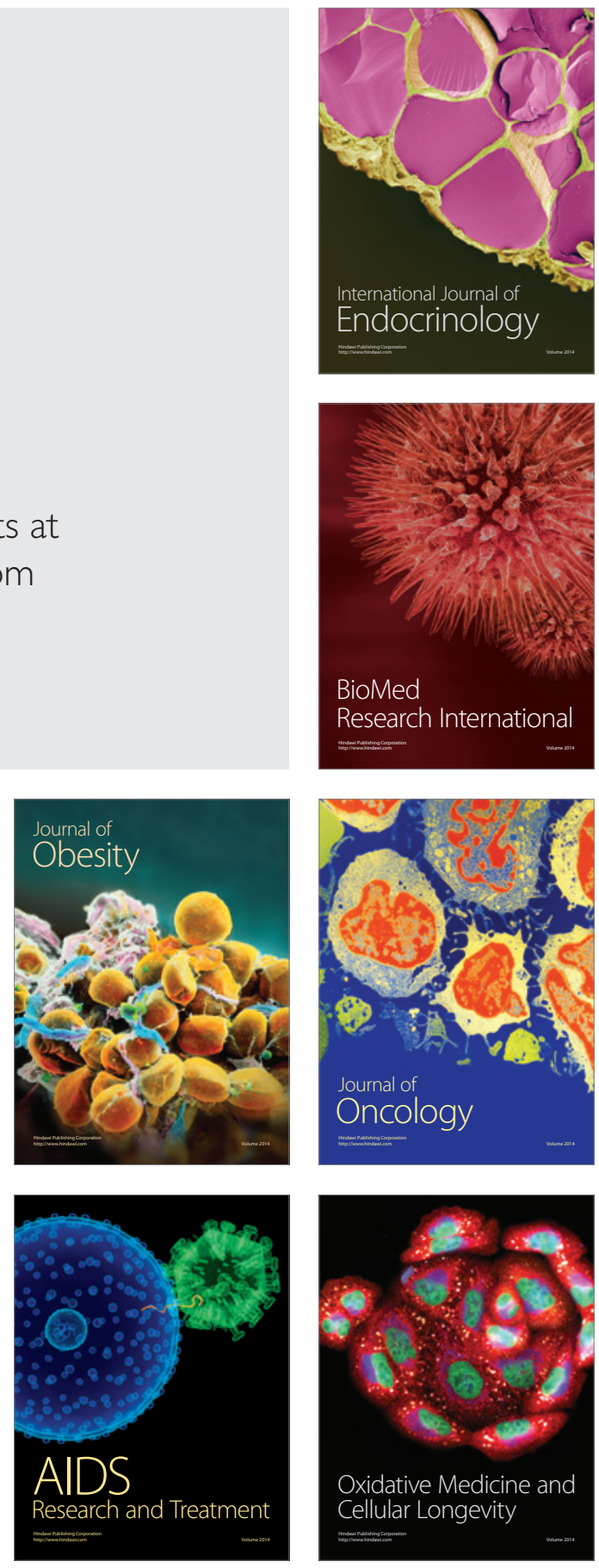